\title{
KOPENAWA Davi et Bruce ALBERT, La chute du ciel. Paroles d'un chaman yanomami
}

José Antonio Kelly Luciani

Traducteur : Philippe Erikson

\section{OpenEdition}

\section{Journals}

Édition électronique

URL : http://journals.openedition.org/jsa/11786

DOI : 10.4000/jsa. 11786

ISSN : $1957-7842$

\section{Éditeur}

Société des américanistes

\section{Édition imprimée}

Date de publication : 5 octobre 2011

Pagination : 339-357

ISSN : 0037-9174

\section{Référence électronique}

José Antonio Kelly Luciani, « kopenawa Davi et Bruce albert, La chute du ciel. Paroles d'un chaman yanomami », Journal de la Société des américanistes [En ligne], 97-1 | 2011, mis en ligne le 10 juillet 2011, consulté le 21 septembre 2020. URL : http://journals.openedition.org/jsa/11786 ; DOI : https:// doi.org/10.4000/jsa.11786

Ce document a été généré automatiquement le 21 septembre 2020.

(c) Société des Américanistes 


\title{
KOPENAWA Davi et Bruce ALBERT, La chute du ciel. Paroles d'un chaman
} yanomami

\author{
José Antonio Kelly Luciani \\ Traduction : Philippe Erikson
}

\section{RÉFÉRENCE}

KOPENAWA Davi et Bruce ALBERT, La chute du ciel. Paroles d'un chaman yanomami, préface de Jean Malaurie, Plon, coll. « Terre Humaine », Paris, 2010, 819 p., bibl., index, gloss., 59 ill. coul. hors-texte, 85 ill. in-texte, cartes

1 Depuis plus d'une dizaine d'années, le bruit courait dans les milieux de l'ethnologie que le tandem Kopenawa-Albert concoctait quelque chose de réellement exceptionnel. Certaines prémices avaient même déjà circulé (Albert 1993; Albert et Kopenawa 2003 ; Viveiros de Castro 2007). L'attente en aura valu la peine ! car voici un opus magnum sans équivalent dans l'anthropologie amazoniste. Nul doute que La chute du ciel... entrera dans le panthéon des grands textes de l'anthropologie et laissera une marque indélébile dans l'histoire de la littérature américaniste.

2 L'ouvrage compte plus de 800 pages et plus d'un millier de notes. Cela, ajouté au fait que l'auteur de ces lignes soit aussi spécialiste des Yanomami (Kelly 2004), justifie que ce compte rendu excède le volume généralement dévolu à ce type d'exercice. Bien qu'écrit sous la forme conventionnelle du rapport de lecture, ce texte se veut, avant tout, un hommage aux auteurs, témoignage de l'immense estime que celui qui tient ici la plume leur porte.

3 À bien des égards, La chute du ciel se présente comme l'inverse de la thèse de Bruce Albert (1985), qui avait pourtant déjà marqué son époque. Il s'agit en effet, ici, non plus d'ethnologie classique, mais d'un projet totalement différent, fruit de la rencontre, à la fin des années 1980, entre deux fortes personnalités unies par une commune volonté de 
défendre le peuple yanomami contre les innombrables ravages que lui faisaient subir les projets de développement brésiliens. Kopenawa, convaincu de la nécessité de délivrer un message qui touche plus directement les Blancs, sollicita Bruce Albert pour l'aider à surmonter le fossé culturel qui l'empêchait jusqu'alors d'élargir son audience occidentale. S'ensuivirent les centaines d'heures d'entretien (plus de mille pages transcrites), mené directement en langue yanomami, pendant plus de dix ans, de 1989 au début des années 2000, et sur lesquelles reposent ce livre. Si «la malencontre historique des Amérindiens avec les franges de notre "civilisation" "(p.17) en constitue la thématique essentielle, ce livre n'en est pas moins aussi, tout à la fois «récit de vie [de Davi Kopenawa], auto-ethnographie et manifeste cosmopolitique » (p. 17).

4 Au sein de la littérature anthropologique, un livre comme celui-ci n'est pas de ceux qui se laissent aisément ranger dans une rubrique précise. En effet, tout en étant le portrait d'un amérindien et de sa communauté, brossé dans une optique dialectique et comparative avec le monde des Blancs, il s'agit aussi, simultanément, d'une critique de la culture occidentale émanant de la communauté des esprits yanomami via l'un de leurs porte-parole : le chamane Davi Kopenawa.

5 Sans doute n'est-il pas non plus excessif de dire que, parmi les très nombreux écrits consacrés aux Yanomami, La chute du ciel représente celui qui, avec un maximum de respect et de méticulosité, a le mieux réussi à dépeindre ce peuple amazonien jusque dans ses moindres détails : de la cosmologie au chamanisme, en passant par la vie quotidienne, la parenté, la guerre, le leadership, les arts oratoires, l'histoire du Contact, l'ethno-politique jusqu'aux conséquences de l'intensification des relations avec l'Étatnation et l'insertion croissante dans une économie mondialisée. Narré entièrement par Kopenawa, La chute du ciel fait tout cela sans aucun recours au jargon académique, rendant l'ouvrage accessible, et même particulièrement attrayant, pour un grand public intéressé par les peuples autochtones et par ce processus aux facettes multiples, présent aux quatre coins de la planète, que l'on nomme aujourd'hui « développement ». Il ne s'agit pas moins d'un livre complexe, qui intéressera au premier chef l'ethnologie et, plus généralement, l'ensemble des sciences sociales. S'il est vrai qu'un des objectifs prioritaires de l'anthropologie est de laisser le champ libre à d'autres formes de construction du sens et de nous éclairer sur d'autres univers conceptuels susceptibles de relativiser le nôtre, alors La chute du ciel est incontestablement un chef d'œuvre anthropologique.

\section{Le pacte ethnographique}

6 "Comprendre une culture autre, c'est faire une expérience sur la nôtre ", disait Wagner $\left(1981\right.$, p. 12) ${ }^{1}$, et cela vaut dans les deux sens. La chute du ciel est un magnifique exemple d'objectivation réciproque, récursive et réflexive, du soi et de l'autre. Le travail créatif accompli respectivement par l'anthropologue et l'amérindien, les textes du premier et les rêves du second, enrichis par un investissement mutuel dans les formes de créativité de l'autre, ont permis de surmonter tous les obstacles du chemin menant du point de départ de toute rencontre ethnographique - là où « leurs méprises sur moi diffèrent de mes méprises sur eux » (ibid., p. 20) ${ }^{2}$ - jusqu'au point de jonction, de reconnaissance et de mise en relation intellectuelle de deux modes de créativité distincts. 
7 Cela fait partie de ce qu'Albert appelle le "pacte ethnographique », supposant un rapport au «terrain» radicalement post-malinowskien, plus impliqué qu'appliqué, pour reprendre les propres termes de l'auteur (Albert 1995 ; 1997). La valeur tout à la fois heuristique et déontologique de ce pacte constitue une des principales leçons à retenir de cet ouvrage, source d'inspiration pour bien des ethnographes dont les terrains respectifs seront certes différents dans la forme, mais pas tant dans le fond, en comparaison avec celui dans lequel Albert s'est construit comme anthropologue. À ses débuts, en 1975, il fut d'emblée et brutalement confronté tout à la fois à une fallacieuse image « exotisante» des "féroces » Yanomami - alors tout juste contactés ${ }^{3}$ - et aux effets déjà tragiques de la construction de la Perimetral Norte - une autoroute destinée à établir une jonction avec la Colombie en coupant à travers le territoire yanomami. D'où l'anxiété initiale de l'auteur, qui se demandait :

Comment concilier connaissance non exotisante du monde yanomami, analyse des tenants et aboutissants du funeste théâtre du "développement » amazonien et réflexion sur les implications de ma présence d'acteur-observateur au sein de cette situation de colonialisme interne ? (p. 568)

Pour en arriver à la recette suivante :

D'abord, bien entendu, rendre justice d'une manière scrupuleuse à l'imagination conceptuelle de mes hôtes, ensuite prendre en compte avec rigueur le contexte sociopolitique, local et global, avec lequel leur société est aux prises et, enfin, conserver une visée critique sur le cadre de l'observation ethnographique ellemême. (pp. 568-569)

Le pacte repose aussi sur la prise de conscience que l'ethnologue n'est en définitive " adopté » par ses hôtes que parce qu'ils espèrent ainsi investir dans l'avenir, faisant le pari qu'il pourra à terme leur servir de médiateur, utilisant ses compétences pour rééquilibrer un tant soit peu l'asymétrie des positions de pouvoir, y compris pour limiter la propagation des épidémies, les spoliations territoriales, les migrations forcées et la myriade d'autres formes de racisme et de discrimination auxquelles les communautés autochtones sont régulièrement confrontées. Un pacte suppose deux parties et l'enjeu consiste à terme, pour les Amérindiens, à :

S'engager dans un processus d'auto-objectification au travers du prisme de l'observation ethnographique, mais sous une forme qui leur permette d'acquérir à la fois reconnaissance et droit de cité dans le monde opaque et virulent qui s'efforce de les assujettir. Il s'agit en retour, pour l'ethnographe, d'assumer avec loyauté un rôle politique et symbolique de truchement à rebours, à hauteur de la dette de connaissance qu'il a contractée, mais sans pour autant abdiquer la singularité de sa propre curiosité intellectuelle (de laquelle dépendent, en grande partie, la qualité et l'efficacité de sa médiation). (p. 571)

Le pacte Kopenawa-Albert nous enseigne que le fameux «engagement» de l'ethnologue, son devoir d'«implication politique», s'accompagne d'une double exigence : respecter l'imaginaire et le style cognitif du peuple qui l'accueille et assumer les responsabilités entrainées par la médiation. Ceux d'entre nous qui ont choisi de passer leur vie dans l'orbite amérindienne savent à quel point les pratiques universitaires et les valeurs académiques tendent à ériger des barrières étanches entre ces deux éléments. D'où, grâce au pacte et par contraste, la splendeur et la force dramatique de La chute du ciel. 


\section{L'écriture}

Dans La chute du ciel, la répartition des rôles respectifs de l'auteur et du narrateur (authorship), loin d'être claire, relèverait plutôt de l'expérimentation. Kopenawa instigateur du projet dont la vie, l'ethnographie et les propos constituent le cœur même de l'ouvrage - semble assumer le statut du narrateur, de l'énonciateur principal. Albert, pour sa part, serait plutôt l'auteur, responsable de l'organisation générale de l'œuvre et du travail de traduction assurant la divulgation de la pensée de Kopenawa à un public élargi. Cependant, au cours de cette tâche particulièrement ardue, Albert choisit de ne pas s'effacer totalement du texte, mais d'y laisser une trace « discrète ", dit-il, de sa présence, un reliquat du travail en collaboration. L'apparat critique - à commencer par les innombrables et fort précieuses notes de fin - éclaire grandement le lecteur, de même que les trois annexes qui permettent de replacer dans leur contexte le peuple yanomami, la région natale de Kopenawa et l'horreur ethnocidaire engendrée par l'orpaillage illégal. L'ouvrage comprend également deux glossaires (ethnobotanique et géographique), plusieurs index, ainsi que des têtes de chapitre et des épigraphes soigneusement choisis ${ }^{4}$. Il est illustré de nombreux dessins yanomami, aussi beaux qu'instructifs, et de nombreuses photographies qui permettent de mettre un visage sur Kopenawa, son peuple et sa trajectoire ${ }^{5}$.

Albert a opté pour une traduction «à distance moyenne», juste, se faufilant habilement entre les écueils du trop littéral et du trop littéraire. On peut le féliciter d'avoir su saisir dans ses moindres détails une rhétorique subtile, qui ne se manifeste généralement, en yanomami, que par le biais de simples suffixes; d'avoir restitué les figures de style typiques du discours yanomami, notamment l'antiphrase (un énoncé négatif destiné à mettre l'accent sur son contraire). Tout cela plonge le lecteur au cœur même du registre poétique yanomami et sonnera particulièrement juste à ceux qui ont quelque familiarité avec les langues amérindiennes. Pour le dire brièvement, même en traduction, les paroles de Kopenawa conservent la flamme métaphorique de la langue yanomami, sa poétique de l'analogie et de l'imagerie sylvestre.

On ne compte plus les histoires d'ethnologues tellement doués pour le travail de terrain qu'ils auraient pu, ou même dû, devenir eux-mêmes indigènes; capables d'accomplir les danses tribales, mais pas de les décrire; volontiers possédés par les esprits indigènes, mais incapables d'en parler. (Schneider in Wagner 1972, p. viii) ${ }^{6}$

13 Ni Kopenawa, ni Albert n'ont succombé à ce travers, en cherchant à devenir Blanc ou Indien, et c'est précisément parce qu'ils se sont constamment efforcés d'appréhender la perspective de l'autre dans une dynamique dialectique que La chute du ciel permet de voir notre "culture scientifique" ou notre "vision matérialiste et marchande du monde " d'un point de vue extérieur: celui d'un chamane et des esprits yanomami. Voilà pourquoi le lecteur qui s'attendrait à des envolées autocritiques postmodernes risque fort d'être déçu, tout comme ceux qui se laissent impressionner par les lénifiants messages pseudo-chamaniques prônant un monde meilleur et le bonheur universel. La chute du ciel est le fruit d'un dur labeur, tout comme Kopenawa a dû travailler dur pour devenir chamane et continue de peiner pour défendre son peuple et son territoire. Kopenawa ne simplifie pas plus sa description du panthéon spirituel yanomami qu'il n'édulcore sa description de l'ethnocide et des dévastations forestières. Faire saisir au lecteur le niveau de complexité et de déséquilibre inhérent aux situations décrites fait partie intégrante des objectifs de ce livre. 
14 Dans le post scriptum, Albert laisse clairement entendre que la réflexion sur l'énonciation (authorship) a été cruciale tout au long de la préparation de l'ouvrage. Viveiros de Castro (2007) a donc parfaitement raison de comparer les stratégies de distanciation narrative qu'on y observe au plus pur des exercices chamaniques; en l'occurrence, sens dessus-dessous, sans doute, mais non moins caractérisé par ces jeux d'enchâssements incessants qui caractérisent le genre. En effet, n'est-ce pas par le truchement d'un Blanc (l'ethnologue) que Kopenawa nous présente le monde de ces derniers, mais tel que se le représenteraient les xapiri (les esprits yanomami)! Difficile, dès lors, de savoir qui, en dernière instance, est l'énonciateur de ces paroles proférées par un chamane qui ne serait lui-même que le porte-voix d'esprits qu'il a appris à connaître grâce aux enseignements de son beau-père. "Ce sont là les paroles d'Omama (le démiurge yanomami)», répète inlassablement Kopenawa. Mais combien d'intermédiaires et combien de traducteurs sont-ils impliqués dans cette construction du sens ? Beaucoup, puisqu'y concourent tout à la fois les xapiri dans leur inépuisable diversité, un maître chamane et son apprenti, ainsi qu'un ethnologue blanc. Et quel est ici l'auditoire? Le message s'adresse clairement aux lecteurs occidentaux. La sagesse qui émane tant de ses exhortations que de sa manière de décrypter le monde des Blancs n'en reste pas moins pertinente pour les proches de Kopenawa, d'autant que beaucoup d'entre eux vivent aujourd'hui dans cet entre-deux hybride où l'Amérindien et le Blanc s'entrelacent.

\section{Kopenawa : philosophe, ethnographe et maître de la métaphore}

Kopenawa est un observateur aussi perspicace qu'insatiable, constamment en quête de connaissances sur sa propre société et d'une inépuisable curiosité à l'égard des motivations et des modes de raisonnement des Blancs. Cet attrait pour le savoir sous toutes ses formes transparaît dans de nombreux passages du livre, témoignant de la propension philosophique de Kopenawa.

Je songe à nos ancêtres qui, au premier temps, se sont transformés en gibier. Je ne cesse de m'interroger : "À quel endroit les êtres de la nuit sont-ils vraiment venus à l'existence ? Comment était le ciel au premier temps ? Qui l'a créé ? Où sont allés les spectres de tous ceux qui sont morts avant nous?» (p. 296)

Je contemplais la forêt blessée et, au fond de moi, je pensais : "Pourquoi leurs machines ont-elles arraché tous ces arbres et cette terre avec tant d'efforts? Pour nous laisser ce chemin de pierres pointues abandonné en plein soleil ? Pourquoi gaspiller ainsi leur argent alors que, dans leurs villes, beaucoup de leurs enfants dorment sur le sol comme des chiens?» (p. 338)

Les Blancs n'ont vraiment aucune sagesse. Ils prétendent que le Brésil est très vaste. Pourquoi viennent-ils alors de toutes parts occuper notre forêt et la dévaster? Chacun d'entre eux n'a-t-il pas déjà une terre, là où sa mère l'a fait naître ? (p. 339)

16 À cet égard, l'ouvrage ne fait que confirmer ce que j'avais déjà constaté en 2008 , lors d'une visite aux communautés yanomami du Venezuela en compagnie de Kopenawa. Dans chacun des villages où nous nous arrêtions, après les interminables réunions pour discuter de politique et de démarcation des terres avec ses pairs de l'autre côté de la frontière, Kopenawa allait trouver les anciens pour s'entretenir avec eux de mythologie et d'histoire, jusqu'à tard dans la nuit et avec une évidente délectation. Cet homme est indéniablement un ethnographe accompli. 
17 Le lecteur sera frappé par l'aisance avec laquelle Kopenawa manie la métaphore et l'incorpore dans les enseignements qu'il dispense avec tant de fougue. Sa maîtrise de la rhétorique impressionnera les lecteurs occidentaux tout comme elle sut conquérir les auditeurs amérindiens. Dans le régime discursif yanomami, c'est la métaphore qui retient l'attention, et Kopenawa est particulièrement habile dans l'art de mettre l'inconnu en résonance avec le connu, de faire surgir des connections inattendues entre la mythologie et les affaires en cours. Il ne suffit pas d'expliquer, il faut aussi divertir, d'où ce constant recours à la poésie et à l'humour. Les quelques exemples qui suivent suffiront à illustrer le talent de Kopenawa à cet égard.

À propos des épidémies qui décimèrent tant de ses aînés au cours de la période initiale des contacts :

En tout cas, il a suffi que nos anciens inhalent cette fumée inconnue pour tous en mourir, comme des poissons qui ignorent encore le pouvoir létal des feuilles du poison de pêche koa axihana. (p. 250)

Au sujet de la capacité des chamanes à rêver - leur voie d'accès au savoir des xapiri :

Nous, en revanche, nous sommes capables de rêver très loin. Les cordes de nos hamacs sont comme des antennes par où le rêve des xapiri descend sans cesse jusqu'à nous. (p. 496)

Sur la déroutante multiplicité des xapiri :

Chaque nom est unique, mais les xapiri qu'il désigne sont innombrables. Ils sont comme les images des miroirs que j'ai vus dans un de vos hôtels. J'étais seul devant eux, mais, en même temps, j'avais beaucoup d'images identiques. (p. 99)

21 Tout au long de l'ouvrage, les rêves et les visions des chamanes (qui donnent accès à une vraie connaissance des images de la forêt et ne sont accessibles ni aux Yanomami ordinaires ni aux Occidentaux) sont régulièrement comparés et contrastés avec l'écriture des Blancs, qui ne savent acquérir le savoir que par le biais de livres et de l'éducation formelle. À l'inverse :

Les paroles des xapiri ne cessent de se rénover et ne peuvent être oubliées [...] elles s'accroissent et se fixent les unes après les autres à l'intérieur de nous et, ainsi, nous n'avons nul besoin de les dessiner pour nous en souvenir. Leur papier c'est notre pensée, devenue, depuis des temps très anciens, aussi longue qu'un grand livre interminable. (p. 554)

22 Sans doute Borgès aurait-il reconnu dans le chamanisme yanomami la concrétisation de son infini livre de sable, qui n'a ni commencement ni fin...

23 Le texte de Kopenawa contient plusieurs formules récurrentes, dont la seule présence renvoie à des significations complexes et qui sont autant de traces de la langue yanomami dans laquelle les propos ont été initialement proférés ${ }^{7}$. Quelques exemples :

24 «C'est ainsi ». Cette interjection introduit des centaines de descriptions détaillées, notamment du monde des esprits yanomami, des choses qui étaient soit surprenantes pour Kopenawa à l'époque, soit de toute évidence trop complexes pour ceux qui ne savent pas voir les esprits et ont donc besoin d'une explication détaillée. À l'instar de la jubilation qu'éprouve le lecteur chaque fois qu'il rencontre «ce n'est pas tout » dans les Mythologiques de Lévi-Strauss, c'est ici à chaque occurrence de "c'est ainsi » qu'on s'agrippe un peu plus fort à l'ouvrage dans l'expectative de développements fascinants sur l'ethnographie yanomami.

«Pas sans raison » : ce livre est, après tout, une histoire de vie et Kopenawa raconte en détail comment il est lui-même passé graduellement de l'ignorance totale du monde 
des esprits yanomami et de celui des Blancs au statut d'apprenti, puis de maitre. Une telle épopée nous révèle que tout a une raison, ou mieux, une histoire. Pour exister "sans raison", des choses ou des événements devraient avoir été produits par la pensée de "personne ». Le lecteur en déduira aisément que rien, en réalité, ne peut être «sans raison ». Très productif dans le discours yanomami, ce "pas sans raison " rappelle aux lecteurs l'humanité immanente de la forêt, leur enseigne qu'un être animé se tient derrière chaque chose et chaque événement, ainsi qu'à l'origine de toute capacité d'affecter ou d'être affecté; il leur donne en somme une leçon d'écologie des relations entre humains et non-humains. Mais l'expression sert aussi à attirer l'attention des lecteurs sur la réelle importance de ce qui semble n'en avoir que peu aux yeux des Blancs; elle suggère une explication alternative, un récit destiné à signaler aux Blancs une connexion qu'ils ignoraient jusqu'alors.

"Des autres gens». Un des thèmes récurrents de ce livre est l'opposition tranchée entre les Yanomami et les Blancs, les premiers étant fils et beau-fils du créateur Omama, les seconds, quoiqu'également créés par le démiurge, s'étant laissé dévoyer par son frère maléfique, Yoasi. Les visions et les rêves chamaniques mènent à une vraie connaissance des images de ce monde, mènent aux paroles des xapiri, aux événements mythiques et à tout ce qui est à l'origine des choses. Le savoir des Blancs, en revanche, incarné par la lecture et l'écrit, est " embrumé », « plein d'oubli ». Ne disposant que des "yeux des esprits des morts", les Blancs ne peuvent pas voir les vraies images du cosmos. Aveugles, ils sont insensibles à l'humanité immanente. Telle est la définition de leur ignorance. La litanie « les Blancs sont des autres gens » me semble révélatrice de l'idée que se fait Kopenawa des Blancs comme bâtisseurs d'un monde ineffable, différent de celui des Yanomami moins en raison de malentendus qui reposeraient sur un socle commun de connaissances partagées, qu'en raison de l'incommensurable divergence d'univers conceptuels radicalement distincts (Viveiros de Castro 2004).

«La valeur de ». S'exprimant en langue yanomami par le simple morphème «në», cette notion renvoie à la valeur et à la trace de quelque chose d'animé. Elle apparaît dans bon nombre des arguments de Kopenawa et semble constituer un élément fondamental de cette politique amérindienne de la nature si particulière, relevant d'une économie politique des gens caractéristique d'un monde peuplé de toutes sortes de "personnes ", tant humaines que non-humaines. Il s'agit là d'une de ces notions totalisantes, qui semblent englober tout ce qui, dans les relations sociales, évoquerait la fertilité, la mortalité, l'échange et la réciprocité. Son apparition dans des contextes très disparates laisse entendre au lecteur qu'il s'agit là d'un concept aussi important qu'« insaisissable », dont les ethnologues auraient tout intérêt à poursuivre l'étude.

\section{Première partie : Devenir autre}

Les huit premiers chapitres de La chute du ciel peuvent se lire comme une série de conférences consacrées à l'ethnographie des esprits. L'étendue de ses connaissances permet à Kopenawa de restituer, avec une acuité éblouissante, la complexité du monde des xapiri. Sa remarquable aptitude à manier la description et l'analogie donnent une consistance inattendue aux entités qui peuplent les différents étages du cosmos et la mythologie. Aucune cosmologie amérindienne n'avait jamais été décrite, jusque dans sa dynamique, avec autant de précision et de clarté. Sur le plan didactique, ce qu'il y a de meilleur est sans doute l'occasion donnée au lecteur de suivre le cheminement 
personnel de Kopenawa, son passage de l'ignorance au savoir, d'une enfance marquée par des rêves lancinants jusqu'à l'initiation chamanique. Avec tous les doutes, les hésitations, les souffrances, les surprises et les déceptions qu'ils impliquent, mais aussi avec cette tenace volonté de savoir, toujours. Comme le formule si bien Wagner (1978, p. 38) «[un] mythe est une "autre culture", même pour les gens de la culture dont il émane ${ }^{8}$. C'est parce que le monde des xapiri est une "autre culture" pour les Yanomami également, que le voyage qu'y fait Kopenawa fait sens pour nous aussi.

Dans ces chapitres, la relation intime entre la chasse et le chamanisme, le perspectivisme, la prédation ontologique et d'autres thèmes classiques de l'ethnologie amazoniste sont traités sur un mode plus auto-descriptif qu'analytique et, superbement, entremêlés avec des scènes de la vie quotidienne des Yanomami, également révélatrices des principes régissant leur sociabilité. On y perçoit aussi très clairement que ce qui tient lieu d'écologie chez les Yanomami relève d'une subtile gestion des relations diplomatiques avec l'ensemble des esprits tapis derrière tout ce qui touche à la forêt, aux humains et au cosmos en général. La météorologie, la fertilité, la dynamique des populations animales, mais aussi des sensations telles la faim et le courage, ou encore des capacités telles celles de penser clairement et de parler de manière convaincante : tout cela trouve son alpha et son oméga chez les êtres animés invisibles. La description que fait Kopenawa de ce "monde d'humanité immanente " est au cœur même de son argumentation, car c'est de là qu'il lance sa critique chamanique de l'objectivation occidentale de la nature et de ses conséquences désastreuses.

Ce que décrit Kopenawa est complexe et on a l'impression qu'il pourrait toujours en rajouter encore. Les xapiri sont minuscules, puissants, d'une aveuglante luminosité, multiples et versatiles. Les xapiri sont grandioses, mais effrayants au début. À certains égards, ils incarnent une version sublimée des valeurs, des pratiques et des usages yanomami, mais ils n'en sont pas moins, en même temps, radicalement différents pour ce qui est de leurs habitudes, goûts, compétences, habitat et autres traits décrits dans les moindres détails.

31 Les miroirs, la luminosité et l'ornementation des xapiri sont des thèmes récurrents, de même que les descriptions des xapiri eux-mêmes: leurs chemins, les miroirs grâce auxquels ils se déplacent, les sols et les toits de leurs maisons, les clairières qu'ils aménagent dans la forêt... Tout ce qui touche aux esprits est orné, brillant et dénué de substance: pures images qui se réfractent inlassablement les unes les autres. Il est aujourd'hui cliché de définir les chamanes comme des "voyageurs de l'espace et du temps ». À lire Kopenawa, on pourrait penser qu'il s'agirait moins de voyages que de manipulations de la dimensionnalité en soi. Le chamanisme serait alors moins une affaire de déplacement à travers des coordonnées spatio-temporelles fixes que la capacité de chambarder les coordonnées elles-mêmes. Réfractions d'images à l'infini, dimensionnalité à géométrie variable, tels sont les rapports introspectifs à l'infini des chamanes yanomami (pour paraphraser Mimica 1988).

Sur la base de fragments de cette ethnographie ayant circulé avant sa parution intégrale, Viveiros de Castro (2007) a déjà formulé quelques commentaires sur la dimension perspectiviste des relations entre xapiri, humains et animaux. Je me contenterai donc de rajouter que le récit de Kopenawa montre aussi que les changements de focale perspectivistes, loin de se cantonner au domaine du visuel, ont des équivalents au niveau acoustique (avec ou sans paroles). S'il est important d'être vu 
par les xapiri, d'attirer leur attention, il ne l'est pas moins d'apprendre à les comprendre, à les écouter et, surtout, à répondre à leurs chants. La beauté et la véracité des chants sont des thèmes récurrents de ce travail, de même que celui de l'obligation d'y répondre si l'on veut acquérir le savoir chamanique. S'il faut certes mourir et devenir soi-même un spectre pour voir exactement comme un xapiri, on peut cependant reproduire leur savoir et leurs chants en acquérant une langue et une gorge comparables aux leurs. La "découverte » du perspectivisme amazonien a surgi d'une étude de glissements de perspectives dans le registre verbal (Viveiros de Castro 1992), même si l'emphase sur le visuel a quelque peu supplanté cette dimension par la suite. Le récit de Kopenawa laisse entendre qu'il y aurait fort à gagner à reprendre la question sous l'angle auditif.

Signalons enfin, et surtout, que le savoir des xapiri et la pratique chamanique sont constamment décrits comme "des visions de loin", des "rêves au long cours", des "paroles anciennes», des "paroles des autres [des xapiri]». Pour connaître, il faut devenir autre. Kopenawa insiste énormément sur ces points, à des fins comparatives, car on trouve là un contraste total avec l'obsession des Blancs pour les livres, leur goût pour la répétition de leurs propres paroles, leur incapacité à ramener des rêves de mondes lointains. Pour Kopenawa, si les Blancs, en dépit de leur indéniable ingéniosité, n'avancent guère, c'est qu'ils sont constamment occupés à se contempler le nombril.

\section{Deuxième partie : La fumée du métal}

Les huit chapitres qui composent cette partie exposent la face la plus sombre de la «malencontre» des Yanomami avec l'expansion étatique brésilienne. Les récits des premiers contacts laissent rapidement la place à une litanie d'épidémies, d'intégrisme évangéliste et d'innombrables morts provoquées par la construction de routes et par l'orpaillage illégal. À cette chronique de l'ethnocide yanomami fait écho l'une des annexes fournies par Albert : un rapport sur le massacre de Haximu, en 1993, au cours duquel seize yanomami furent tués par des garimpeiros (orpailleurs clandestins).

Chaque chapitre débute par une ou plusieurs épigraphes (coupures de presse, commentaires de généraux de l'armée ou de missionnaires des New Tribes Mission, dorénavant NTM), qui illustrent le point de vue de certains Blancs sur ce que décrit ensuite le texte: les différents épisodes du Contact et la subséquente invasion du territoire yanomami. Le lecteur peut ainsi se faire une idée du fossé colossal qui sépare la souffrance des Yanomami et l'arrogante rhétorique du progrès.

L'influence des Blancs sur le mode de pensée yanomami est une thématique constante de ce texte.

Nos anciens aimaient leurs propres paroles. Ils étaient vraiment heureux aussi. Leur esprit n'était pas fixé ailleurs. Les propos des Blancs ne s'étaient pas introduits parmi eux [...]. Ils possédaient leurs propres pensées, tournées vers leurs proches. (p. 223)

Il était important d'agir en ayant toujours quelqu'un, un parent, en tête (voir Surrallès 2003). Une partie de ce à quoi les choses "sans raison " s'opposent représente les produits de la pensée de quelqu'un. Le commentaire de Kopenawa est à cet égard poignant car, çà et là, on perçoit qu'anciennement et de nos jours encore, le contact avec les Blancs a égaré les Yanomami, les entraînant à trop penser aux étrangers, à leurs objets manufacturés, à leur mode de vie. Le message concerne au premier chef 
cette frange du public de Kopenawa, composée de Yanomami qui intègrent volontiers des éléments de la culture occidentale à la leur, mais tâtonnent encore pour savoir quelle en serait la dose optimale.

L'histoire de Kopenawa lui-même est également de celles qui se situent à l'intersection des mondes yanomami et occidental. Du temps des premiers contacts, ceux qui sont venus habiter avec lui à Watoriki, où il réside aujourd'hui, ont été décimés par une terrible épidémie. Bien des années plus tard, alors qu'il vivait à $\mathrm{T}^{\mathrm{h}}$ oot ${ }^{\mathrm{h}} \mathrm{ot}^{\mathrm{h}} \mathrm{opi}$ avec des missionnaires des NTM, une seconde vague d'épidémie tua la majorité de ses proches parents, y compris sa mère. Kopenawa jette un regard rétrospectif sur la façon dont ses anciens succombèrent à la force de séduction des objets manufacturés, vecteurs de ces fumées invisibles qui, selon les théories étiologiques yanomami, étaient responsables des épidémies qui venaient si souvent les exterminer. Sans doute le plus triste est-il encore de constater qu'il ne s'agit pas uniquement d'images lointaines d'un passé révolu. La situation sanitaire des Yanomami demeure, depuis lors, en état de crise quasi permanente.

Les rites funéraires yanomami sont destinés à effacer toute trace du défunt. Ils servent également à aviver la soif de vengeance des parents survivants. Kopenawa laisse clairement entendre que sa propre soif de défendre son peuple puise sa source dans le souvenir de ses propres parents dévorés par les épidémies xawara apportées par les Blancs 9 .

La trajectoire de Kopenawa se poursuit avec le récit de sa vie auprès des missionnaires des NTM. S'y mêlent l'histoire d'une ébauche de conversion individuelle et collective qui ne dépassa jamais vraiment la phase expérimentale avec, en contrepoint, le récit détaillé de l'entêtement obsessionnel avec lequel les membres du NTM voulaient imposer la parole de Dieu, s'attaquer au chamanisme et, plus généralement, perfectionner les pratiques ethnocidaires si répandues dans toute l'Amazonie. Il est d'ailleurs intéressant, à ce propos, de relever qu'une des raisons de l'échec de la conversion des Yanomami semble liée à l'impossibilité de voir Dieu ou d'entendre ses réponses : précisément des éléments présentés dans la première partie comme critères essentiels de légitimation du savoir lié aux xapiri. En définitive, Dieu semble avoir été incapable de protéger son troupeau des épidémies à $\mathrm{T}^{\mathrm{h}}$ oot ${ }^{\mathrm{h}} \mathrm{ot}{ }^{\mathrm{h}}$ opi : carence rédhibitoire puisque la religiosité yanomami ne laisse aucune place à la foi.

41 Après les épidémies de $\mathrm{T}^{\mathrm{h}}$ oot $^{\mathrm{h}} \mathrm{ot}^{\mathrm{h}} \mathrm{opi}$, Kopenawa s'est trouvé pour ainsi dire totalement dénué de proches parents. À l'échelle yanomami, cette absence de liens sociaux signifiait l'extrême misère, ce qui rend la saga de cet homme d'autant plus remarquable. Rongé de solitude, Kopenawa commence à se distancier de sa communauté. Son travail pour la FUNAI (l'agence gouvernementale brésilienne en charge des affaires indiennes) lui donne l'occasion de visiter d'autres zones du territoire yanomami, de se familiariser avec les terres des Blancs, de passer du temps dans les villes de Boa Vista, Manaus et même Iauareté, à la frontière colombienne, en service chez les Maku. Durant cette période, employé intermittent de la FUNAI sous différentes administrations, il exerce bon nombre d'emplois: on lui demande de localiser les communautés les plus éloignées, de faire l'interprète, d'apprendre les soins infirmiers, d'installer des postes de la FUNAI, et même de nettoyer des piscines à Manaus ! À cette époque, il est lui-même tenté d'essayer de devenir Blanc, mais surtout, il s'initie à la micro-politique amazonienne. Il apprend à décrypter la manière dont les différents agents gouvernementaux et non gouvernementaux cherchent à orienter le 
futur des Yanomami. Il se familiarise avec cette arène politicienne faite d'accusations réciproques et révélatrice des différents intérêts qui pèsent sur le territoire, des entreprises minières aux militaires soucieux de souveraineté nationale, sans oublier les ONG venues les aider. C'est aussi la période où il entend pour la première fois parler de " démarcation des terres ", une cause qui marquera durablement son existence, jusqu'à aujourd'hui.

Cette gamme élargie d'expériences lui permet de prendre conscience de l'ampleur de la dévastation engendrée par les projets de développement brésiliens, lui donnant une vision d'ensemble et une connaissance directe des effets délétères de la brutalité liée aux travaux de voierie et à l'orpaillage sauvage. Deux cents kilomètres de route furent construits, lacérant tout le territoire yanomami, et la ruée vers l'or débridée qui, à la fin des années 1980, entraîna plus de 40000 garimpeiros dans la région, entraîna la mort d'environ $10 \%$ de la population amérindienne, pour ne rien dire des dégâts environnementaux et de la destruction sociale.

$\mathrm{Au}$ cœur de cette crise, Kopenawa décide de changer de vie. Il veut devenir chamane et, pour ce faire, se plie aux exigences de l'apprentissage chamanique avec une détermination aussi farouche que celle qu'il mettra ultérieurement au service de la défense de son peuple. Il bénéficie de l'aide, d'une part, de son beau-père Lourival grand homme et chamane, généreux et accueillant, que l'auteur de ces lignes a eu l'occasion de rencontrer à Watoriki-, d'autre part, du groupe de militants indigénistes à l'origine du CCPY, ONG fondée entre autres par Bruce Albert et dont l'action sera déterminante pour l'obtention de la démarcation des terres yanomami.

C'est donc avec un regard de chamane que Kopenawa analyse les événements dramatiques qui se déroulent sous ses yeux. Voyant un lien entre la dévastation locale et les processus socio-économiques globaux, les chamanes de Watoriki élaborent une théorie de l'histoire et des motivations des Blancs qui débouche sur une annonce prophétique, émanant des xapiri : la chute du ciel - cataclysme déjà survenu à l'horizon mythique - se reproduira, écrasant aussi bien les Yanomami que les Blancs, si ces derniers ne cessent pas de consommer la forêt, d'en retirer le pétrole, l'or et autres minéraux qu'Omama, dans sa grande sagacité, avait cachés sous terre. En «cuisant " ces matériaux extraits du sous-sol dans les usines qui produisent les biens matériels auxquels ils sont viscéralement attachés, les Blancs brûlent la poitrine du ciel, répandent des fumées xawara et propagent ainsi encore plus d'épidémies.

Dans l'économie politique radicalement différente qui est la leur, rien n'a plus de valeur que les gens, et la terre est plus solide encore que la vie:

Toutes les marchandises des Blancs ne seront jamais suffisantes en échange de tous ses arbres, ses fruits, ses animaux et ses poissons. Les peaux de papier de leur argent ne seront jamais assez nombreuses pour pouvoir compenser la valeur de ses arbres brûlés, de son sol desséché et de ses eaux souillées [...]. Aucune marchandise ne pourra acheter tous les Yanomami dévorés par les fumées d'épidémie. Aucun argent ne pourra rendre aux esprits la valeur de leurs pères [chamans] morts ! (p. 373) 


\section{Troisième partie : La chute du ciel}

Eiffel et dans le Bronx dont il nous rend compte avec le plus de détails. L'ambition indéniablement universaliste du discours chamanique ressort clairement de ces pages, où l'on voit Kopenawa resituer chacun de ces lieux et les expériences qu'il y a vécues dans la topographie symbolique des mythes relatifs à la création et à l'histoire des Blancs, tout en acquérant des connaissances supplémentaires sur les esprits qui résident parmi eux. De tels périples au loin comportent en outre beaucoup de risques, car les chamanes ne doivent en aucun cas se rendre là d'où viennent leurs auxiliaires spirituels, sous peine d'en mourir. Kopenawa dut déployer des trésors d'ingéniosité pour contourner cette difficulté, encore renforcée par le risque bien réel que ses esprits laissés à Watoriki ne le quittent à tout jamais, frustrés qu'ils pourraient être par l'abandon de leur père absentéiste.

50 Les musées semblent systématiquement heurter la sensibilité des populations autochtones. Les Yanomami éliminent systématiquement toute trace de leurs morts et les exposer à la vue de tous dans un édifice public relève littéralement, pour Kopenawa, de l'inconcevable. Mais surtout, il se demande si tel est vraiment l'avenir que lui réservent les Blancs. Est-ce cela qui restera de nous quand ils en auront fini avec la 
forêt ? Les voyages de Kopenawa ont, sans conteste, enrichi la mythologie yanomami. Dans cette optique, les voyages outre-mer relèvent toujours un peu de la prédiction.

Dans le Bronx, Kopenawa est frappé par l'indifférence sereine des Blancs face à l'exclusion sociale :

Pourtant, si au centre de cette ville [New York] les maisons sont hautes et belles, sur ses bords, elles sont en ruine. Les gens qui vivent dans ces endroits n'ont pas de nourriture et leurs vêtements sont sales et déchirés. Quand je me suis promené parmi eux, ils m'ont regardé avec des yeux tristes. Cela m'a fait peine. Ces Blancs qui ont créé les marchandises pensent qu'ils sont ingénieux et valeureux. Pourtant, ils sont avares et ne prennent aucun soin de ceux qui, parmi eux, sont dépourvus de tout. Comment peuvent-ils penser être de grands hommes et se trouver aussi intelligents? Ils ne veulent rien savoir de ces gens misérables qui font pourtant partie des leurs. Ils les rejettent et les laissent souffrir seuls. Ils ne les regardent même pas et se contentent, de loin, de leur attribuer le nom de «pauvres ». (p. 460)

Pour Kopenawa, l'intelligence des Blancs s'investit essentiellement dans la technique, et leur culture est avant tout matérielle. Comme la plupart des autres Yanomami de ma connaissance, il est donc totalement affligé par la portion congrue accordée à la parenté par les Blancs, certes ingénieux mais nettement infra-sociaux.

À de multiples reprises, Kopenawa se demande si le génie manufacturier des Blancs est vraiment aussi malin qu'il le paraît. L'un des exemples d'anthropologie comparative les plus intéressants se trouve dans un chapitre justement nommé "L'amour de la marchandise ", qui détaille le contraste entre la tendance des Blancs à assujettir les relations sociales à l'accumulation des biens, d'une part, et la propension inverse des Yanomami à faire circuler les objets, d'autre part. Sa réflexion porte, entre autres, sur la catégorie indigène de matihi, qui inclut les ornements personnels des Yanomami, les gourdes dans lesquelles sont conservées les cendres des morts et aussi, depuis l'arrivée des Blancs, leurs biens matériels. Kopenawa tient, à cet égard, des propos aussi profonds que beaux :

C'est ainsi. Les marchandises ne meurent pas. C'est pourquoi nous ne les accumulons pas de notre vivant et nous ne les refusons jamais à ceux qui les demandent. Si nous ne les donnions pas, elles continueraient à exister après notre mort et moisiraient seules, délaissées sur le sol de notre foyer. Elles ne serviraient alors qu'à faire peine à ceux qui nous survivent et pleurent notre mort. Nous savons que nous allons disparaitre, c'est pourquoi nous cédons facilement nos biens [...]. Ainsi les marchandises nous quittent-elles rapidement pour se perdre dans les lointains de la forêt avec les hôtes de nos fêtes reahu ou de simples visiteurs [...]. Lorsqu'un être humain meurt, son spectre n'emporte aucun de ses biens sur le dos du ciel, même s'il est très avare! Les objets qu'il avait fabriqués ou acquis sont abandonnés sur la terre et ne font que tourmenter les vivants en ravivant la nostalgie de sa présence. Nous disons alors que ces objets sont orphelins et que, marqués par le toucher du mort, ils font peine. (p. 435)

Il est ironique que tant de violence ait été imposée aux Yanomami au nom de leur prétendue violence. Il est bien connu que l'image guerrière qu'on leur a imputée a été instrumentalisée par les gouvernements militaires brésiliens et les gens désireux de s'approprier leur territoire, y voyant la légitimation de son invasion au nom de sa mise au service de la productivité et du soi-disant développement. Le fameux ouvrage de Chagnon (1968), maintes fois réédité et présentant les Yanomami comme des "gens féroces ", n'aura guère non plus contribué à leur bien-être. Kopenawa semble, à ce sujet, résolu à rétablir la vérité. Et tout comme il l'a fait à propos des biens matériels, il insiste sur un contraste: ici, celui entre les raids de vengeance des Yanomami et les 
guerres des Blancs. Les premiers, modestes et motivés par le désir d'apaiser la rage provoquée par le décès d'un proche, les secondes démesurées, visant à l'accumulation de biens matériels dans des proportions absurdes, et entraînant une quantité pharamineuse de morts, sans commune mesure avec les règlements de compte interindividuels des Amérindiens. Ici encore, ce sont les gens qui comptent, et non le pétrole, les minerais et autres biens qui suscitent la convoitise des Blancs et les incitent à faire la guerre ${ }^{10}$. Tant les motivations que les proportions sont incommensurables :

Eux se bataillent en très grand nombre, avec des balles et des bombes qui brûlent toutes leurs maisons. Ils tuent même les femmes et les enfants ! Et ce n'est pas pour venger leurs morts, car ils ne savent pas les pleurer comme nous le faisons. Ils font leur guerre simplement pour de mauvaises paroles, pour une terre qu'ils convoitent ou pour y arracher des minerais et du pétrole. (p. 474)

Kopenawa ne sous-estime pas les différents moyens ritualisés dont usent les Yanomami pour résoudre leurs conflits, y compris les raids guerriers. Il ne s'agit cependant que de moyens de contrôler l'accumulation de rage. Clastres voyait la "société primitive", cette "totalité une», comme une machine à segmenter, interdisant l'émergence d'instances de pouvoir séparées de la société. Dans la même veine, Kopenawa insiste lui aussi sur la mise à l'écart de la rage, évitant ainsi une accumulation de colère prolongée qui aboutirait à des guerres sur une large échelle. Il estime cependant qu'aujourd'hui, le courage des Yanomami doit être intégralement dirigé contre leurs véritables ennemis : les Blancs qui veulent dévorer leurs terres et leur peuple. En dépit de tous les dommages subis, Kopenawa prend cependant bien garde d'éviter les généralisations abusives :

Nous ne sommes pas les ennemis des Blancs. Mais nous ne voulons pas qu'ils viennent travailler dans notre forêt car ils sont incapables de nous rendre la valeur de ce qu'ils y détruisent. C'est ce que je pense. (p. 372)

L'ouvrage s'achève sur l'un des avertissements les plus vifs proférés par Kopenawa, appelant solennellement à empêcher les Blancs de détruire la planète et faire disparaître les chamanes, seuls capables de prévenir l'imminente chute du ciel :

Sans chamans, la forêt reste fragile et ne tient pas en place toute seule [...]. Si les êtres de l'épidémie continuent à y proliférer, les chamans finiront par tous mourir et plus personne ne pourra l'empêcher de tourner au chaos. Maxitari, l'être de la terre, Ruëri, celui du temps couvert et Titiri, celui de la nuit, se mettront en colère. Ils pleureront leur mort et la forêt deviendra autre. Le ciel se couvrira de nuages obscurs et le jour ne se lèvera plus. Il n'en finira pas de pleuvoir. Un vent d'ouragan soufflera sans trêve. La forêt ne connaîtra plus le silence [...]. Cela est déjà arrivé, mais les Blancs ne se demandent jamais pourquoi [...]. La terre se gorgera d'eau et commencera à se putréfier. Puis les eaux la recouvriront peu à peu et les humains deviendront autres, comme c'est arrivé au premier temps. (pp. 534-535)

57 Lorsque le ciel tombera, nous serons tous écrasés et envoyés nous écrabouiller dans l'inframonde. Les chamanes yanomami le savent, l'ayant déjà vu au commencement des temps :

Si les Blancs finissaient par devenir plus avisés, mon esprit pourrait retrouver le calme et la joie. Je me dirais : «C'est bien! Les Blancs ont acquis de la sagesse. Ils ont enfin pris en amitié la forêt, les êtres humains et les esprits xapiri!». Mes voyages prendraient fin. J'aurais bien assez parlé loin de ma maison et empli de peaux de papier du dessin de mes paroles. Je n'irai plus visiter la terre des Blancs que de temps à autre. J'y dirais alors à mes amis : « Ne m'appelez plus si souvent ! Je veux devenir esprit et continuer à étudier avec les xapiri! Je veux seulement 
devenir plus savant!». Je me cacherais alors dans la forêt avec mes anciens pour boire la yãkoana jusqu'à en redevenir très maigre et oublier la ville. (p. 527)

Espérons que La chute du ciel soit prochainement traduite, notamment en anglais, en espagnol et en portugais. Les paroles de Kopenawa pourront alors se propager, comme antidote, avec autant de force et de rapidité que les épidémies cannibales xawara. Ce n'est qu'en améliorant la pensée des Blancs que les effets délétères de leurs actes pourront être contrés. Kushu ha!

\section{BIBLIOGRAPHIE}

\section{ALBERT Bruce}

1985 Temps du sang, temps des cendres. Représentation de la maladie, espace politique et système rituel chez les Yanomami du Sud-Est (Amazonie brésilienne), thèse de doctorat, université de Paris-X Nanterre, Nanterre.

1993 « L'or cannibale et la chute du ciel. Une critique chamanique de l'économie politique de la nature », L'Homme, 126-128, pp. 35-70.

1995 « Anthropologie appliquée ou “anthropologie impliquée” ? », in Jean-François Baré (éd.), Les applications de l'anthropologie : un essai de réflexion collective depuis la France, Karthala, coll. « Hommes et sociétés », Paris, pp. 87-118.

1997 «"Ethnographic situation" and ethnic movements. Notes on Post-malinowskian Fieldwork », Critique of Anthropology, 17 (1), pp. 53-65.

ALBERT Bruce et Davi KOPENAWA

2003 Yanomami. L'esprit de la forêt, Actes Sud/Fondation Cartier pour l'art contemporain, Paris.

\section{CHAGNON Napoléon}

1968 Yanomamö. The fierce people, Holt, Rinehart and Winston, New York.

\section{HANBURY-TENISON Robin}

1982 Les Aborigènes de l'Amazonie. Les Yanomami, Éditions du Club France-Loisirs/Time Life, Paris.

KELLY José Antonio

2004 Relations within the health system among the Yanomami in the Upper Orinoco, Venezuela, PhD. dissertation, University of Cambridge, Cambridge.

LÉVI-STRAUSS Claude

1984 Paroles données, Plon, Paris.

MIMICA Jadran

1988 Intimations of infinity : the mythopoeia of the Iqwaye counting system and number, afterword by Roy Wagner, Berg Publishers, Oxford.

SURRALLÉS Alexandre

2003 Au cœur du sens : perception, affectivité, action chez les Candoshi, Éditions du CNRS, Paris. 
VIVEIROS DE CASTRO Eduardo

1992 From the enemy's point of view : humanity and divinity in an Amazonian society, University of Chicago Press, Chicago.

2004 « Perspectival anthropology and the method of controlled equivocation », Tipiti, 2 (1),

pp. 3-22.

2007 « La forêt des miroirs. Quelques notes sur l'ontologie des esprits amazoniens ", in Frédéric B. Laugrand et Jarich G. Oosten (éds), La nature des esprits dans les cosmologies autochtones, Les Presses de l'Université Laval, Québec, pp. 45-74.

WAGNER ROY

1972 The curse of Souw : principles of Daribi clan definition and alliance in New Guinea, University of Chicago Press, Chicago.

1978 Lethal speech : Daribi myth as symbolic obviation, Cornell University Press, Ithaca.

1981 The invention of culture, University of Chicago Press, Chicago.

\section{NOTES}

1. «[E]very understanding of another culture is an experiment with our own » [Toutes les notes ont été ajoutées par le traducteur, Philippe Erikson (Université Paris Ouest Nanterre La Défense)].

2. "[T]heir misunderstanding of me was not the same as my misunderstanding of them ».

3. «Indiens agressifs et guerriers ", «tribu indienne où aucun Blanc n'avait jamais pénétré », en disait par exemple la jaquette d'un autre ouvrage publié, en 1968, dans ce que Jean Malaurie appelle, dans l'auto-élogieuse préface qu'il donne à ce livre, « la bibliothèque indienne de Terre Humaine » (p. 7). En 1982, l'introduction d'un livre pourtant remarquablement bien documenté et signé par le fondateur de Survival International présentait encore les Yanomami comme le "groupe le plus important de tous les Indiens des Amériques à vivre dans un isolement quasitotal [...] ethnie coupée du monde et aux origines lointaines [...] au mode de vie demeuré pratiquement immuable depuis des milliers d'années » (Hanbury-Tenison 1982, p. 5).

4. Les deux tout premiers incipits de l'ouvrage (pp.13-15) sont respectivement des citations de Claude Lévi-Strauss et de Davi Kopenawa. Non sans humour, le rapprochement entre les deux est encore renforcé par le titre choisi juste après (pp.37-40) pour les propos introductifs de Kopenawa: "paroles données", qui évoque évidemment l'ouvrage éponyme de Lévi-Strauss (1984).

5. Les cahiers photos de l'ouvrage comprennent essentiellement des clichés de Kopenawa pris entre 1972 et 2009. Il apparaît le plus souvent peint, soit chez lui, soit en compagnie des grands de ce monde, tels le secrétaire général des Nations Unies et pas moins de trois présidents du Brésil. On le voit aussi devant des lieux emblématiques de sa trajectoire cosmopolite : la Tour Eiffel, l'Empire State Building, le parlement britannique, Stonehenge, etc.

6. "Legions are the stories of anthropologists who are such magnificent fieldworkers that they actually went native, or should have, who could do the tribal dances but not describe them, who could become possessed by the native spirits but not discuss them ".

7. Dans son avant-propos, Bruce Albert précise par ailleurs que «la transcription des onomatopées, pourtant si savoureuses et finement codifiées en yanomami, a été limitée au maximum afin d'alléger le texte. En revanche, quelques interjections, employées de façon récurrente pour introduire des propos cités, ont été conservées. Il s'agit de : asi ! qui indique la colère ; awe! qui marque l'approbation; haixopë! qui dénote la réception (approbatrice) d'une information nouvelle; ha! qui marque la surprise (satisfaite et/ou ironique); hou! qui dénote 
l'irritation; ma! qui exprime la désapprobation et, enfin, oae! qui marque une remémoration subite $»($ p. 26).

8. "[a] myth is "another culture", even for those of its own culture".

9. Sur le concept de xawara, ainsi que le rapport entre fumées, Blancs et épidémies, la note 8 du chapitre 7 de La chute du ciel... précise : «l'épidémie (xawara) se propage dans le monde visible sous forme d'une fumée (xawara wakëxi). Aux yeux des chamans, elle prend la forme d'une cohorte d'esprits maléfiques cannibales (xawarari) semblables aux Blancs qui dévorent et cuisinent leurs victimes ».

10. Les propos de Kopenawa sur les conflits des Blancs ont été recueillis juste après la première guerre du Golfe (1990-1991), qui a visiblement eu beaucoup d'influence sur son point de vue.

\section{AUTEURS}

\section{JOSÉ ANTONIO KELLY LUCIANI}

Universidade Federal de Santa Catarina, Florianópolis 